\title{
Revisión del material cerámico de la colección Pastaza (Amazonía ecuatoriana)
}

Geoffroy de Saulieu

\section{(2) OpenEdition \\ Journals}

Edición electrónica

URL: https://journals.openedition.org/jsa/3208

DOI: $10.4000 /$ jsa. 3208

ISSN: 1957-7842

Editor

Société des américanistes

\section{Edición impresa}

Fecha de publicación: 1 junio 2006

Paginación: 279-301

ISSN: 0037-9174

\section{Referencia electrónica}




\title{
REVISIÓN DEL MATERIAL CERÁMICO \\ DE LA COLECCIÓN PASTAZA \\ (AMAZONÍA ECUATORIANA)
}

\author{
Geoffroy De SAULIEU *
}

A Pedro Porras
Sit terra tibi levis

En 1969, el arqueólogo Pedro Porras emprendió una misión en la región del río Huasaga, un afluente del Pastaza, en la provincia amazónica de MoronaSantiago en el Ecuador (Figura 1). A su retorno trajo consigo diversos materiales cerámicos que presentaban un estado de conservación muy variable. Dichos materiales fueron publicados en 1975 bajo el nombre de « fase Pastaza ». Porras (1975) distinguió 9 tipos cerámicos: Pastaza Blanco sobre Rojo, Pastaza Corrugado, Pastaza Decorado con Uñas, Pastaza Exciso, Pastaza Falso Corrugado, Pastaza Inciso y Punteado, Pastaza Inciso Retocado, Pastaza Rojo, Pastaza Rojo Inciso. En la misma publicación, Porras (ibid., p. 135) presentó 4 fechas radiocarbónicas comprendidas entre 2883 a.C. y 1437 d.C. No obstante, estas fechas plantean problemas, puesto que implican una continuidad cultural excepcionalmente larga. Si seguimos a Porras, la «fase » Pastaza sería en parte contemporánea de una de las más antiguas culturas agro-alfareras que se conocen en la costa ecuatoriana, Valdivia, y de las antiguas fases del sitio ceremonial de Santa Ana-La Florida en la provincia de Zamora-Chinchipe, situada sobre la vertiente suroriental de la cordillera de los Andes ecuatorianos (Valdez et al. 2005). Por este motivo, a este material cerámico algunos lo han denominado « el Valdivia de la Amazonía » (cita en Porras 1987b, p. 116). Tres fragmentos poco representativos figuran en el catálogo de la exposición Ancient Ecuador dedicada al Formativo ecuatoriano y organizada por Lathrap en Chicago en 1975 (Lathrap, Collier y Chandra 1975, p. 109, $\left.\mathrm{n}^{\circ} 584,585,586\right)$. En cuanto a Meggers (1987), integró la cerámica Pastaza dentro de su Horizonte Achurado Zonal con el mismo título que la cerámica peruana de Waira-Jirca y de Tutishcaynio. A partir de esto, la

* Chercheur associé à l'unité de recherche 092 de l'IRD « Les adaptations humaines aux environnements tropicaux durant l'Holocène ", IRD Quito, Whymper 442 y Coruña, apartado potal 1712 857, Quito, Équateur [saulieu@freesurf.fr].

Journal de la Société des Américanistes, 2006, 92-1 et 2, pp. 279-301. (O) Société des Américanistes. 


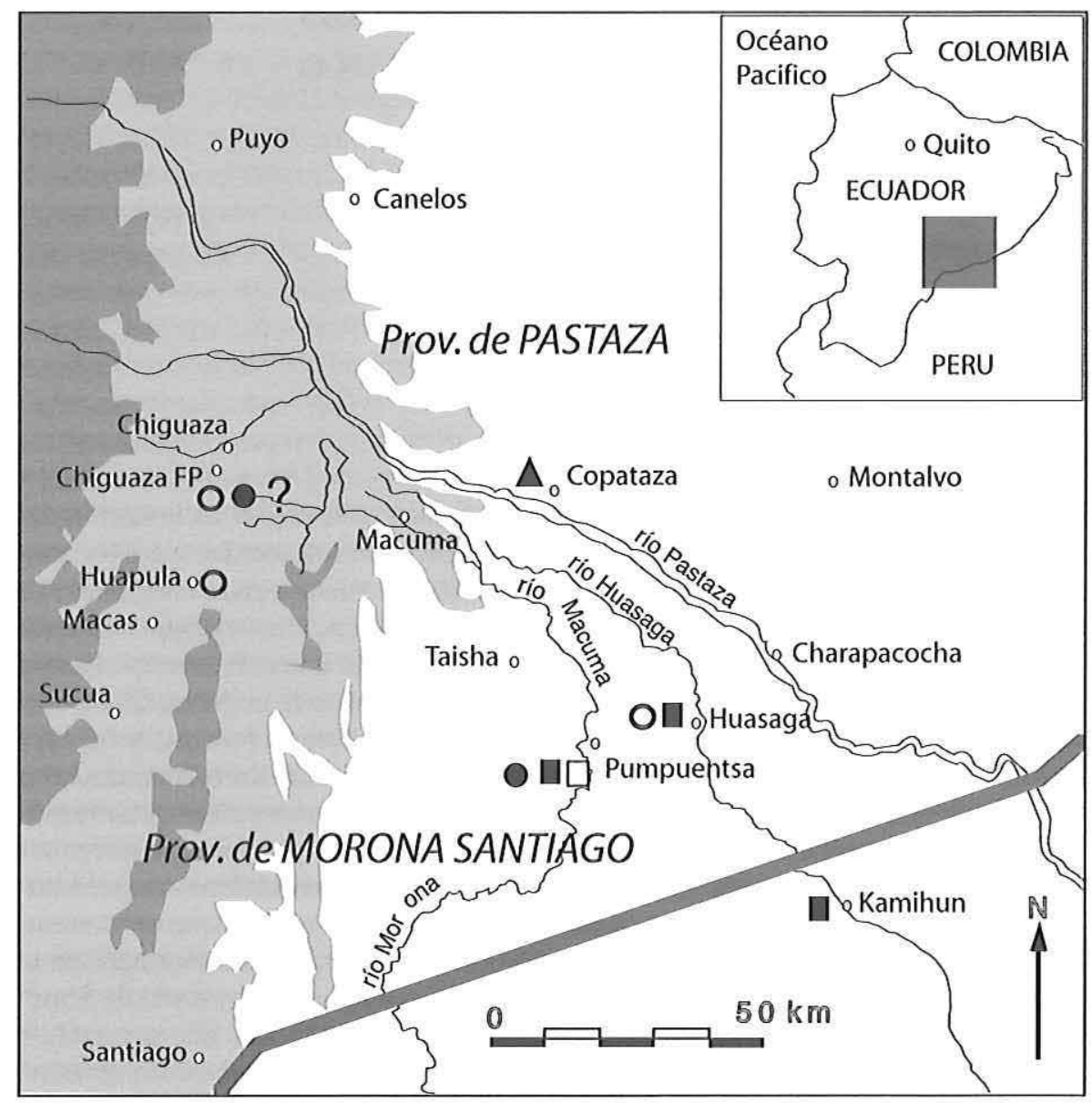

FIG. 1 - Mapa de los principales sitios mencionados en el texto

El mapa también ubica:

- con cuadrados negros, los sitios que contienen material Pastaza, tipo Pastaza-Kamihun;

- con cuadrado blanco, los sitios que contienen material Pastaza, tipo Pastaza-Kamihun, con motivos figurativos;

- con triángulo, el sitio de Copataza de donde proviene el cuenco conservado en el Museo Arqueológico y Etnográfico de Puyo.

- con círculos, los sitios que contienen material corrugado. 
«fase Pastaza » figuró sistemáticamente en las cronologías del Ecuador, que hayan sido ellas publicadas por arqueólogos o por instituciones culturales oficiales como el Banco central. En el extranjero, esta fase también se volvió un hecho poco discutido: muy recientemente Lumbreras (2005, p. 116) citó la « fase Pastaza » como representativa de unos agricultores de principios del Formativo dentro de la región amazónica.

Un material semejante al Pastaza se dio a conocer en la misma época en que Pedro Porras publicó el suyo. En 1975, De Boer (1975; De Boer et al. 1977) señaló un material proveniente de las riveras del Huasaga, costado peruano que él denominó « Kamihun », muy similar al « Pastaza Inciso y Punteado » descrito por Porras. Por esta razón designaremos este estilo como Pastaza-Kamihun.

Posteriormente, en 1980, Stephen Athens realizó una trinchera en el sitio de Pumpuentsa, a $20 \mathrm{~km}$ al oeste del sitio excavado por Porras (Figura 1). En la estratigrafía reportada había material Pastaza y se obtuvieron fechas radiocarbónicas las cuales arrojaron una edad comprendida entre los siglos II a.C. y VIII d.C. (Tabla 1). Para Athens $(1984 ; 1986)$, y a pesar de la importante separación de las dos fechas, la fase Pastaza entraría en el periodo de Desarrollo regional. Sin embargo, algunos problemas subsisten: por un lado, no se explica la importante diferencia entre las dos dataciones y, por el otro, estratigráficamente están invalidas, puesto que la más reciente proviene del nivel 5 y la más antigua de carbones dispersos entre los niveles 5 y 4; finalmente cabe notar que el tipo llamado « achurado zonal », el cual caracteriza aparentemente los niveles más profundos, también aparece en las capas superiores.

Durante el verano del 2004, en el Museo Weilbauer de la Universidad Católica de Quito, Patricio Moncayo me permitió revisar algunos archivos y examinar la colección epónima de la « fase Pastaza » proveniente de los sondeos y prospecciones realizados por Porras en Huasaga. La revisión efectuada de las dos series de datos fue demasiado rápida como para rechazar rotundamente el trabajo de Porras, pero tampoco permite ir claramente en el sentido de los resultados de Stephen Athens. Lo que nos quedó claro, sin embargo, es la diversidad del material cerámico que forma esta colección. Existen verdaderos conjuntos distintos (grupos de cerámica que comparten características comunes por lo que respeta a la tecnología utilizada en su elaboración y, también, con relación al acabado de superficie, Echeverría 1981, pp. 94-95), los cuales pueden compararse con materiales de la colección del Museo de Arqueología y Etnografía de Pastaza en la ciudad de Puyo (colección no publicada que reúne cerámica proveniente de las riveras del río Pastaza); detectamos también posibles correlaciones con lo descrito en un informe inédito de investigaciones realizadas en otras localidades de la provincia de Morona Santiago (Echeverría et al. 1996). 


\section{AnOtaciones PRELIMINARES}

En la publicación de 1975, Pedro Porras menciona diez sondeos (de los cuales nueve fueron realizados a proximidad de una base militar localizada al borde del río Huasaga), así como también numerosas prospecciones en el sector. La colección depositada en la Universidad Católica de Quito conserva las huellas de esta diversidad: muchos tiestos no numerados corresponden probablemente a recolecciones superficiales de prospecciones, mientras que el material numerado de manera discontinua entre 410 y 463 , corresponde probablemente a los sondeos y a la profundidad en la cual se recogió (se utilizaron niveles artificiales de $10 \mathrm{~cm}$, pero ellos fueron numerados de manera poco comprensible). Limité mi revisión del material a los tiestos numerados con el fin de evitar los riesgos de mezclas con otras colecciones constituidas por Porras.

El sitio denominado « Huasaga » por Porras, se encuentra a lo largo del río del mismo nombre a $300 \mathrm{~m}$ sobre el nivel del mar. Se encuentra sobre una terraza aluvial la cual domina unos 15 metros el cauce del río y tiene aproximadamente $300 \mathrm{~m}$ de largo y $50 \mathrm{~m}$ de ancho. Porras hizo nueve sondeos: dos entre la base militar que se encontraba allí y el río (sondeos 1 y 2), y siete al oeste de la pista aérea de los militares (sondeos 3, 4, 5, 6, 7, 9, 10). Un último sondeo, el 8, fue efectuado a $3 \mathrm{~km}$ de la terraza. Los sondeos son descritos brevemente (el 4 aún no ha sido descrito), observándose que, por lo general, no rebasan los $30 \mathrm{~cm}$ de profundidad (sondeos 1, 2, 3, 5, 6, 7, 8); el 9, por su parte, álcanzó $40 \mathrm{~cm}$ de profundidad. En cuanto al sondeo 10 tuvo $90 \mathrm{~cm}$ de profundidad: según el autor habría sido realizado " en el centro de una pequeña colina artificial ». La estratigrafía descrita en la página 79 de la publicación de 1975 distingue 7 capas: " detritos vegetales », « tierra negra » pobre en tiestos (29 $\mathrm{cm}$ de espesor), « tierra negra, con algunos tiestos » (20 cm de espesor), " tierra negra abundante en tiestos » (30 cm de espesor), «tierra negra » donde « disminuyen los tiestos» (15 cm de espesor), « tierra amarillenta » con pocos tiestos (15 cm de espesor), y « tierra amarilla estéril ». Sin embargo, los espesores indicados no concuerdan con las profundidades que figuran en la tabla de conteo del material presentada en la página 114: les sobrepasan por casi $20 \mathrm{~cm}$.

De acuerdo con el croquis publicado, también se subraya que el sondeo $10 \mathrm{se}$ situó a un extremo de la pista de un campo de aviación. Porras obtuvo de este sondeo seis fechas radiocarbónicas contradictorias, de las cuales publicó únicamente dos; las otras fechas (dos realizadas en 1973 y dos en 1976) fueron encontradas en los archivos durante el verano del 2004 (Tabla 1).

Dadas las fechas contradictorias de este sondeo 10, así como la gran diversidad del material, es probable que Porras excavó sin darse cuenta la tierra desplazada con ocasión de la construcción de la pista aérea. Además, el sondeo 10 , como todos los otros sondeos, presenta un material muy diverso desde todos 


\begin{tabular}{|c|c|c|c|c|c|}
\hline $\begin{array}{c}\text { Número } \\
\text { de } \\
\text { datación }\end{array}$ & Sitio & $\begin{array}{l}\text { Situación } \\
\text { estratigráfica }\end{array}$ & $\begin{array}{l}\text { Datación } \\
\text { BP } \\
\text { No } \\
\text { calibrada }\end{array}$ & $\begin{array}{l}\text { Dataciones } \\
\text { Calibradas a } 2 \text { sigmas } \\
\text { (según el método de } \\
\text { Stuiver et al. 1998) }\end{array}$ & Referencia \\
\hline DIC 798 & Pumpuentsa & Nivel 5 & $1210+1-65$ & $\begin{array}{l}690-704 \text { AD } \\
1260-1246 \mathrm{BP}\end{array}$ & Athens 1984 \\
\hline $\begin{array}{l}\text { Beta } \\
22015\end{array}$ & Pumpuentsa & Nivel 4-5 & $1910+/-110$ & $\begin{array}{c}150-134 \mathrm{BC} \\
(2099-2083 \mathrm{BP})\end{array}$ & Athens 1986 \\
\hline ISGS-384 & Huasaga & $\begin{array}{l}\text { Sondeo 1, } \\
10-20 \mathrm{~cm} \text {. }\end{array}$ & $600+/-65$ & $\begin{array}{c}1295-1437 \mathrm{AD} \\
(655-513 \mathrm{BP})\end{array}$ & Porras 1975 \\
\hline I-9157 & Huasaga & $\begin{array}{l}\text { Sondeo } 6 \text {, } \\
10-20 \mathrm{~cm} .\end{array}$ & $819+/-90$ & $\begin{array}{l}1029-1311 \mathrm{AD} \\
(921-639 \mathrm{BP})\end{array}$ & Porras 1975 \\
\hline SI-1363 & Huasaga & $\begin{array}{l}\text { Sondeo } 10 \text {, } \\
20-30 \mathrm{~cm} \text {. }\end{array}$ & $740+l-95$ & $\begin{array}{l}1057-1087 \mathrm{AD} \\
(893-863 \mathrm{BP})\end{array}$ & $\begin{array}{l}\text { (1973) no } \\
\text { publicado }\end{array}$ \\
\hline I-9157 & Huasaga & $\begin{array}{l}\text { Sondeo } 10 \text {, } \\
30-40 \mathrm{~cm} \text {. }\end{array}$ & $810+/-90$ & $\begin{array}{c}1035-1145 \\
(915-805 \mathrm{BP})\end{array}$ & $\begin{array}{l}\text { (1976) no } \\
\text { publicado }\end{array}$ \\
\hline I- 9159 & Huasaga & $\begin{array}{l}\text { Sondeo } 10, \\
60-70 \mathrm{~cm}\end{array}$ & $4000+/-100$ & $\begin{array}{c}2864-2806 \mathrm{BC} \\
(4813-4755 \mathrm{BP})\end{array}$ & Porras 1975 \\
\hline 1-9158 & Huasaga & $\begin{array}{l}\text { Sondeo } 10 \text {, } \\
70-80 \mathrm{~cm} .\end{array}$ & $4000+l-100$ & $\begin{array}{c}2864-2806 \mathrm{BC} \\
(4813-4755 \mathrm{BP})\end{array}$ & $\begin{array}{l}\text { (1976) no } \\
\text { publicado }\end{array}$ \\
\hline ISGS-385 & Huasaga & $\begin{array}{l}\text { Sondeo } 10 \text {, } \\
70-80 \mathrm{~cm} .\end{array}$ & $4155+/-75$ & $\begin{array}{c}2883-2557 \mathrm{BC} \\
(4832-4506 \mathrm{BP})\end{array}$ & Porras 1975 \\
\hline SI-1364 & Huasaga & $\begin{array}{l}\text { Sondeo } 10 \text {, } \\
80-90 \mathrm{~cm} \text {. }\end{array}$ & $895+l-65$ & $\begin{array}{l}1029-1267 \text { AD } \\
(921-683 \mathrm{BP})\end{array}$ & $\begin{array}{l}\text { (1973) no } \\
\text { publicado }\end{array}$ \\
\hline
\end{tabular}

TABLA 1 - Dataciones publicadas e inéditas (en negrillas) de sitios pertenecientes a la « fase Pastaza ». Abreviaciones: Beta = Beta Analytic; ISGS = III. State Geological Survey; $\mathrm{I}=$ Teledyne Isotope; $\mathrm{SI}=$ Smithsonian Institution; $\mathrm{DIC}=$ DICARB Radioisotope Company.

los puntos de vista, lo que podría eventualmente ser interpretado como el resultado de una mezcla. Si intentamos reconstruir las estratigrafías por los números de lotes cerámicos, no se observa ningún orden, ni tendencia general: algunos sondeos presentan una repartición homogénea del material sin importar la profundidad, mientras que otros dan órdenes de aparición de los diferentes tipos cerámicos totalmente contradictorios. Esta impresión se hace aún más evidente cuando vemos que los tiestos se encuentran generalmente bastante fragmentados y erosionados, sin posibilidad de ser pegados, lo que podría sugerir que el material ha sido desplazado y redepositado por el río Huasaga. Ello nos señala que los conjuntos, tipos y seriaciones propuestos por Porras en su publicación, deben ser tomados con prudencia.

Al finalizar la revisión rápida del material, nos pareció imprescindible insistir sobre la diversidad cerámica contenida en esta colección, antiguamente publicada como una fase homogénea compuesta de varios tipos. Y eso cuanto más que no es raro encontrar mención de la « fase Pastaza » en la arqueología ecuato- 
riana, en particular para designar, entre otras cosas, la cerámica con decoración « corrugada ».

\section{DiVERSIDAD DEL MATERIAL Y COMPARACIONES}

La colección constituida por Pedro Porras es extremadamente variada. No obstante, la ausencia de datos estratigráficos detallados y de archivos de los registros constituye un grave limitante. Por otra parte, de 5,688 tiestos colectados inicialmente, hoy en día únicamente subsisten 1,513. Tal vez los 4,175 tiestos faltantes correspondan a aquellos no diagnósticos que Porras habría suprimido a finales de los 1970. De todas formas esto dificulta mucho la comparación entre nuestra observación y lo publicado por Porras. De allí que nos enfocaremos en algunos conjuntos singulares bien reconocibles. Nuestro trabajo, preliminar, busca facilitar estudios posteriores más completos, notablemente al destacar elementos de comparación con varios sitios del pie de monte andino y de la alta Amazonía peruano-ecuatoriana.

\section{Cerámica Pastaza-Kamihun: 740 tiestos}

Cuatro categorías de pastas se encuentran en este conjunto; ellas se diferencian ante todo por el grosor del desgrasante. La pasta más fina representa la mayoría de los tiestos (688 tiestos) y presenta una consistencia bastante homogénea. Es de color gris a negro, y tiene una textura delicada conteniendo partículas muy finas, inferiores a $1 \mathrm{~mm}$. También se pueden distinguir granos brillantes o blanquecinos y partículas rosadas. La segunda pasta está representada por 34 tiestos. Es de color gris, marrón y negro, y es prácticamente idéntica a la precedente, distinguiéndose por el grosor del desgrasante que es netamente mayor $(>1 \mathrm{~mm})$. Las otras dos pastas son más gruesas y sólo se reconocen en menos de veinte tiestos. El espesor medio de estos tiestos es homogéneo y se sitúa entre los 0,4 y $0,6 \mathrm{~cm}$.

La casi totalidad de los tiestos provienen de cuencos, y unos pocos de tecomates. Se ha detectado únicamente un plato y no hay presencia de ollas. Entre los 129 bordes que pueden ser orientados y medidos, identificamos cuatro grandes clases de cuencos, con labios simples redondeados o en media ojiva. Dominan las formas abiertas: la primera clase está conformada por 77 bordes de cuencos abiertos (Figura 2.1), la segunda por 23 bordes que presentan una abertura más o menos equivalente al ancho máximo situado a nivel de la panza, pero con un perfil exterior de paredes convexo (Figura 2.2); la tercera forma corresponde a 21 fragmentos de recipientes con abertura ligeramente cerrada (Figura 2.3); finalizando, la cuarta la conforman 8 bordes que presentan aberturas netamente más restringidas que el ancho máximo de la panza (aquí figuran algunos tecomates) (Figura 2.4). 

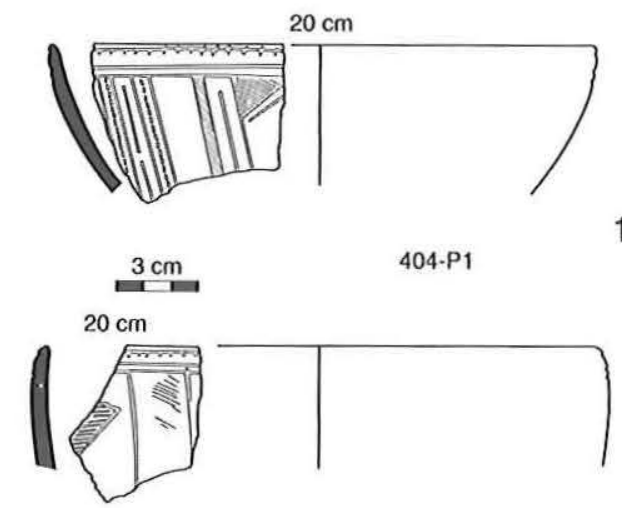

1.
$3 \mathrm{~cm}$
403-5
2.

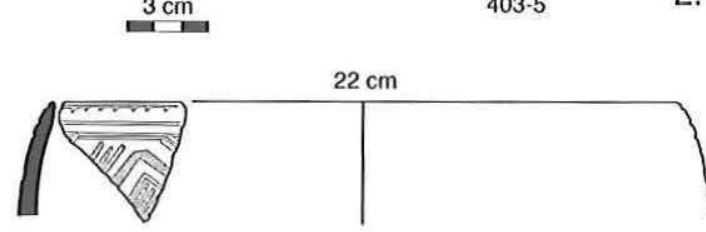

$3 \mathrm{~cm}$

$462-1$

3.

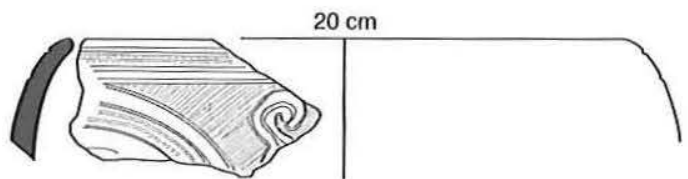

$3 \mathrm{~cm}$

460.P2

밀

4.

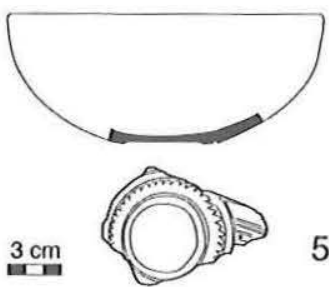

FIG. 2 - Tiestos Pastaza-Kamihun

1. Borde de cuenco abierto;

2. Borde de cuenco con abertura aproximadamente equivalente al ancho máximo del recipiente;

3. Borde de cuenco ligeramente cerrado;

4. Borde de cuenco claramente cerrado;

5. Base cóncava de cuenco. La reconstrucción de la parte superior es hipotética. 
Dentro del conjunto se observó que las formas son extremadamente estereotipadas: las paredes no son rectas, ni verticales (aun en el caso de la clase 2), pero siempre convexas. Los diámetros de abertura son poco variables: oscilan entre 20 y $24 \mathrm{~cm}$ en las tres primeras formas (sin embargo los extremos tienen respectivamente 16 y $28 \mathrm{~cm}$ de diámetro), y entre 12 y $20 \mathrm{~cm}$ para la forma más cerrada. Hemos reconocido una sola base plana. Casi todas las demás bases identificables (26 de 29) son cóncavas y corresponden a un tipo bastante frecuente de decoración con incisiones y círculos concéntricos alrededor de la parte central la cual forma casi una base anular (Figura 2.5). En la colección se han visto sólo 2 bases convexas redondeadas, pero tal vez eran más numerosas antes de ser eliminados $\operatorname{los} 4,175$ tiestos faltantes.

No se encontraron ollas en la muestra. Estos recipientes fueron sin duda realizados en otras pastas y estilos.

Las técnicas utilizadas para la decoración son poco numerosas: incisión sobre pasta fresca, excisión, punteado, achurado, pulido externo zonal, interior enteramente pulido. Generalmente los tiestos decorados presentan la mayoría de estas técnicas utilizadas al mismo tiempo. Sin embargo, algunos, cuya decoración es más sencilla, presentan únicamente incisiones sobre pasta fresca con o sin pulido externo zonal, o incisiones sobre pasta fresca asociadas con achurado y/o con punteado sin pulido externo zonal. Sólo un fragmento muestra restos de pigmentos rojos en el interior de las incisiones, tal como los tiestos del sitio más meridional de Kamihun, en el lado peruano de la frontera. Esta modalidad decorativa fue probablemente frecuente en el sitio Huasaga; sin embargo, es posible que los tiestos hayan sido arrastrados y lavados por la acción del río. Además, al parecer Porras utilizó tiza para resaltar las incisiones de los tiestos, lo que impide una buena observación del fondo de los surcos. Los motivos privilegian netamente las formas geométricas con ángulos. No obstante, algunos motivos curvos, en algunas ocasiones circulares, aparecen sobre la panza. La estructura de la decoración es suficientemente repetitiva como para que se distinga una organización estándar. Hay tres registros: el primero cubre de 3 a $5 \mathrm{~cm}$ bajo el labio; un segundo registro, mediano, ocupa la parte central de la panza; y el último registro se sitúa entre el registro mediano y la base. Dentro del primer registro, encontramos una sucesión de líneas horizontales incisas de las que parten muescas, perpendiculares o no (Figuras 2.1, 2.2, 2.3, 2.4). Más abajo, dentro del registro mediano, aparecen motivos geométricos angulares, a veces remplazados por achurados o punteados cerrados muy finos, produciendo planos decorativos (Figuras 2.1, 2.3 y 2.4). Generalmente, dentro de estas anchas zonas achuradas o punteadas, pueden aparecer formas independientes, curvas y en espiral en sus extremidades (Figura 2.4). Los conjuntos de motivos repetitivos, compuestos de líneas paralelas de puntos y de grecas, forman bandas dispuestas verticalmente u oblicuamente sobre la panza (Figura 2.1). A veces, estas decoraciones se complican y describen ellas mismas meandros geométricos con encru- 
cijadas y grecas. Ellas terminan frecuentemente sobre las decoraciones que rodean la base cóncava, las cuales están constituidas por una serie de líneas paralelas, de las cuales usualmente una está acompañada de muescas perpendiculares (Figura 2.5).

El Pastaza-Kamihun forma un conjunto que se afirma fuertemente desde un punto de vista estilístico: tiene un número limitado de formas (cuencos y tecomates), pero se caracteriza por una inversión decorativa impresionante. Una rápida comparación con los datos disponibles en otros sitios donde se encuentra material semejante, sugiere que la colección de Porras corresponde a una de las variantes de un conjunto Pastaza mucho más amplio. En efecto, en el sitio de Pumpuentsa, el Pastaza tiene algunas formas iconográficas figurativas, lo que constituye una diferencia con los sitios de Huasaga y Kamihun que presentan decoraciones únicamente abstractas. Además, en Pumpuentsa, la cerámica Pastaza-Kamihun está asociada con una variante más simple llamada por Stephen Athens « achurado zonal ». Ésta se caracteriza por formas tanto de cuencos como de ollas. La decoración es el resultado «de la alternancia de bandas estrechas de zonas achuradas con zonas lisas » (Athens, 1986), la cual está realizada de manera mucho más burda y simple que en el Pastaza-Kamihun. También se distingue la presencia frecuente de una línea incisa en el interior del recipiente justo por debajo del labio (Figura 3.2). Esta decoración está totalmente ausente en Huasaga y Kamihun (De Boer et al. 1977), sitios que presentan fuertes semejanzas entre sí. Se debe señalar que el tiesto de la figura 3.2 típico de lo que Athens llama " achurado zonal» y que está expuesto en el Museo Amazónico de la Universidad Salesiana de Quito, tendría una procedencia muy occidental, tal vez en el valle de Chiguaza (sitio Chiguaza F.P.), es decir, al pie de los Andes. Finalmente, el único recipiente entero conocido proviene de Copataza y muestra la existencia de una variante septentrional al privilegiar un registro central ancho, totalmente cubierto de impresiones puntuales, dentro del cual se encuentran formas geométricas y curvilíneas. El resultado decorativo parece más simple desde el punto de vista de la estructura de la decoración, aunque, desde el punto de vista visual, tiene la misma carga de decoraciones clásicas que las que han sido encontradas en otros sitios (Figura 3.1). Así, el Pastaza-Kamihun sería una variante oriental de un conjunto general Pastaza caracterizado por incisiones principalmente geométricas sobre pasta fresca, el cual incluiría también dos variantes occidentales presentes en Pumpuentsa y una variante septentrional presente en Copataza. Vistas la calidad de la pasta y las decoraciones cerámicas del conjunto Pastaza, éste pudo haber sido una vajilla especial desde el punto de vista de la identidad. La distribución espacial de los sitios comprende un territorio que se encuentra entre el río Pastaza en el Norte y al Este, el alto Morona, sus afluentes (en particular el Macuma) y el alto Huasaga al Oeste. 

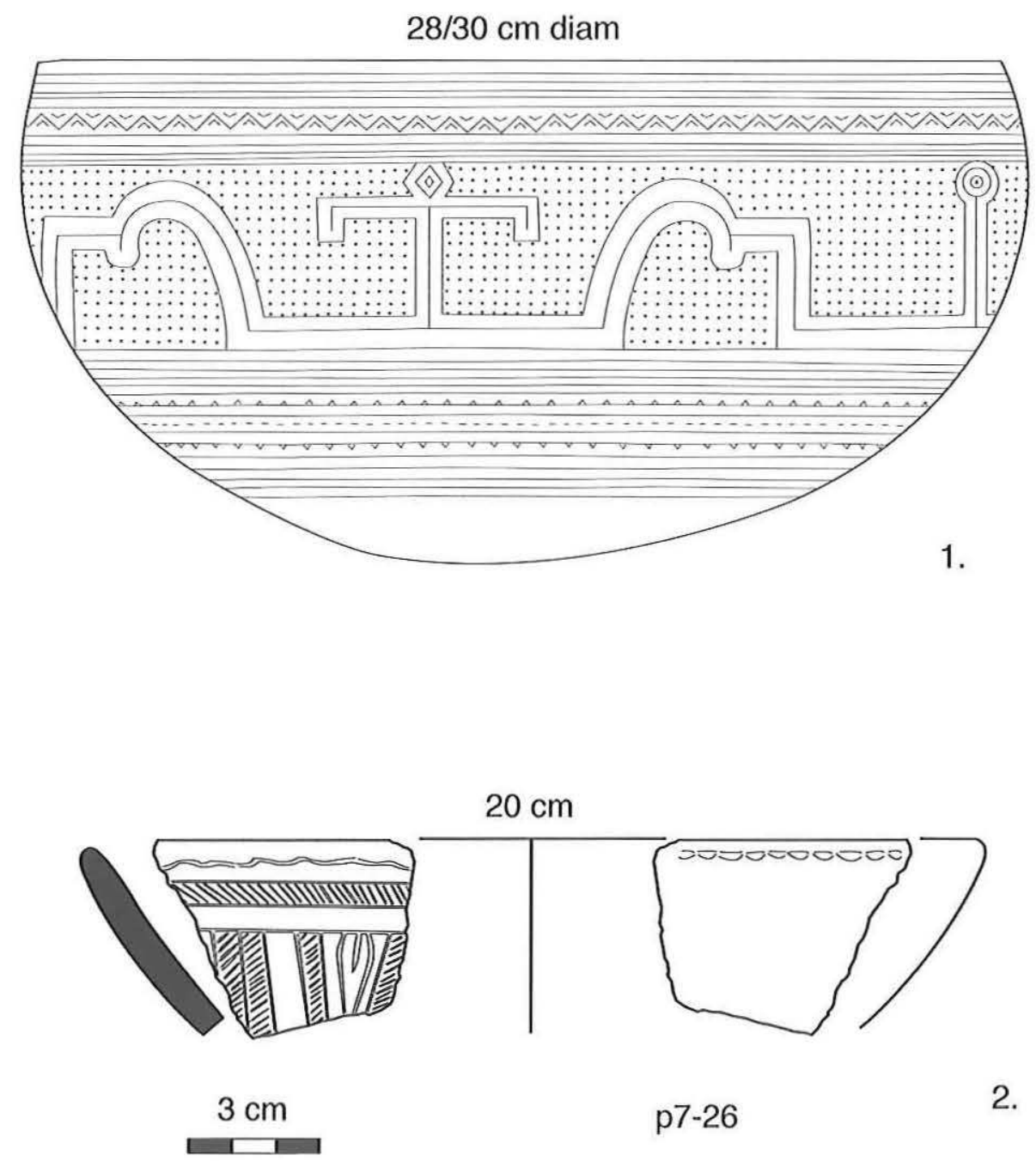

FIg. 3 - Variante Pastaza

1. Bosquejo de un recipiente proveniente de Copataza, expuesto en el Museo Etnográfico y Arqueológico de Puyo.

2. Borde de cuenco tipo « achurado zonal », tal como lo define Athens (1984; 1986). Museo Amazónico de la Universidad Salesiana de Quito. 


\section{Cerámica incisa y excisa con engobe rojo: 89 tiestos}

Este conjunto de tiestos erosionados incluye cuatro pastas diferentes. La primera presenta una textura arcillosa y contiene un desgrasante constituido por granos brillantes y oscuros, generalmente inferiores a $1 \mathrm{~mm}$, así como una pequeña cantidad de chamota. Esta pasta está presente en 58 de los 88 tiestos que pertenecen a esta familia. Por otro lado, se aprecia una variante muy gruesa en 9 tiestos (las partículas sobrepasan $1 \mathrm{~mm}$, e incluso $2 \mathrm{~mm}$, y constituyen más del $50 \%$ de la pasta). Originalmente, el desgrasante llevó partículas vegetales (paja). A continuación se encontraron 16 tiestos con una pasta de color crema a beige, y matices grises. También se pueden reconocer partículas blanquecinas y brillantes inferiores a $1 \mathrm{~mm}$, además de gránulos rojos que podrían ser chamota. También se distinguen gránulos negros. En general la textura es arenosa pero fina. Finalmente, 6 tiestos parecen constituir una variante de la pasta precedente, pero diferente por el corte y la calidad de las partículas arenosas que son más gruesas y numerosas. El espesor de los tiestos varía entre 0,4 y $0,8 \mathrm{~cm}$.

Las formas son poco variadas: de un total de 89 tiestos, 81 provienen de cuencos, 1 de una olla, y 7 tiestos no son identificables a causa de su estado de conservación. Este conjunto cuenta 21 bordes que pueden ser orientados y medidos (no se ha encontrado ninguna base), entre los cuales distinguimos cuatro grupos que se diferencian claramente del conjunto Pastaza-Kamihun: el primero corresponde a cuencos abiertos (12 ejemplares) (Figura 4.1), el segundo a cuatro tiestos provenientes de cuencos cuyas paredes tienden a ser verticales, más o menos rectilíneas (Figura 4.2); el tercero corresponde a cuatro fragmentos de cuencos que van de poco a muy cerrados (Figura 4.3), y el cuarto contiene un fragmento de olla.

Las técnicas decorativas utilizadas son las siguientes: incisión, excisión (ahondamiento de determinadas áreas de la superficie de la vasija, quitando parte de la arcilla cuando todavía esta fresca, a fin de obtener motivos en plano contrastado) y achurado. Éstas fueron realizadas sobre tiestos con engobe rojo-anaranjado. Así mismo, los motivos observados son seis: 1/ espiral con exterior inciso y/o exciso (Figura 4.4), 2/ triángulos excisos (Figura 4.5), 3/ figuras triangulares compuestas con el exterior inciso y/o exciso (Figura 4.6), 4/ círculos incisos dispuestos en medallones bajo el borde (Figura 4.7), 5/ líneas paralelas incisas, 6/ dos motivos subtriangulares achurados dispuestos perpendicularmente (Figura 4.8). Es necesario mencionar la existencia de motivos hasta ahora no identificables en razón de la fragmentación del material. Podemos decir que, en este conjunto, existe una tendencia general de formas decorativas curvilíneas, espirales y geométricas.

Las decoraciones son en realidad bastante variadas, y dan una impresión de heterogeneidad. Con todo, se debe especificar que el material de este conjunto de la colección de Porras está fragmentado y erosionado, lo cual dificulta la asocia- 

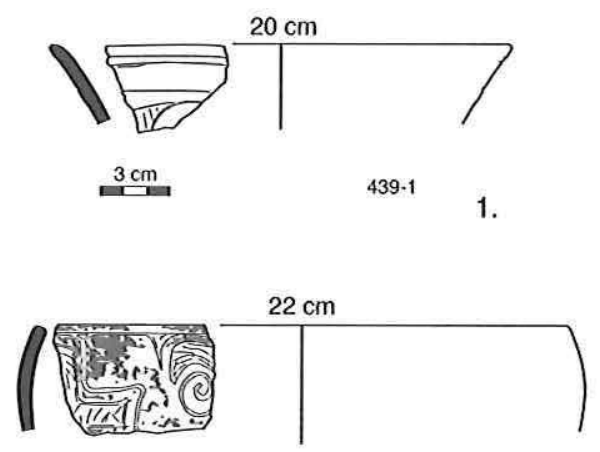
$3 \mathrm{~cm}$

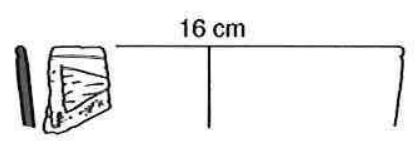

$3 \mathrm{~cm}$

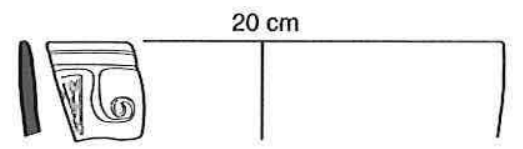

403-10

2.

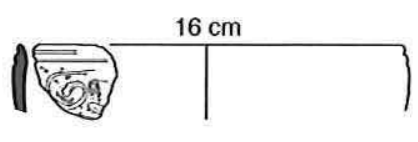

$3 \mathrm{~cm}$

4.

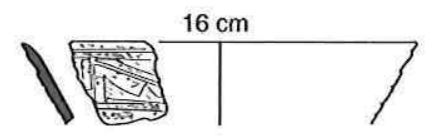

$3 \mathrm{~cm} \quad 422 \cdot \mathrm{Pl}^{2}$

6.
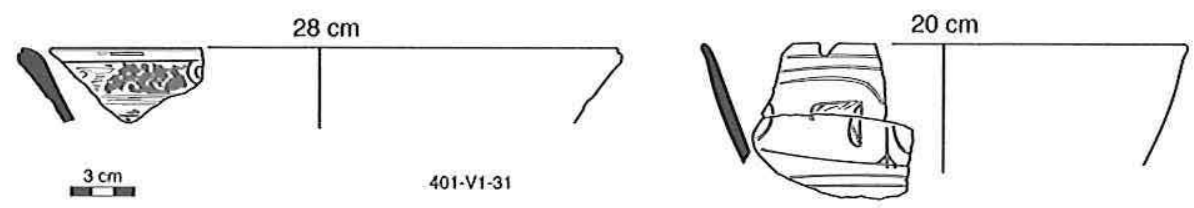

7.

$3 \mathrm{~cm}$

423-18

8.
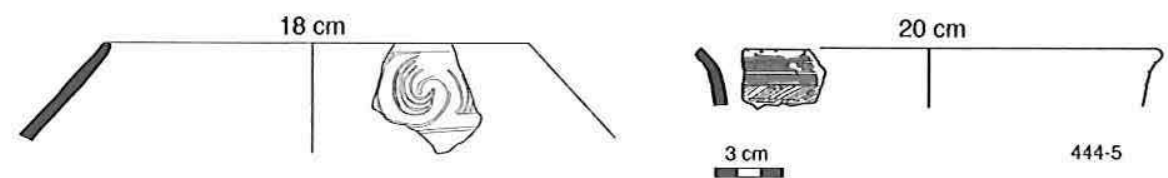

$3 \mathrm{~cm}$

9.

10.

FIG. 4 - Tiestos incisos-excisos con engobe rojo 
ción entre las diferentes decoraciones. Sin embargo, una comparación con la colección del Museo de Puyo avoca a favor de la unidad de este material, puesto que en ello los tiestos están menos fragmentados y mejor conservados. Dicha colección permite de hecho hacer asociaciones entre diferentes motivos y diferentes técnicas. Además, en Puyo, los tipos de pastas son poco diversos. Esta comparación también nos permitió reagrupar los tiestos muy fragmentados y erosionados de la colección de Pedro Porras.

Otra comparación es posible con el material llamado «rojo inciso » (Figura 4.9) descubierto en la provincia de Morona Santiago, cerca de Macuma, a $70 \mathrm{~km}$ al noroeste del sitio Huasaga (Echeverría et al. 1996, Fig. 6). El área de repartición de esta cerámica es entonces bastante amplia. Sin embargo, dicha cerámica no es abundante en el sitio Huasaga, y está ausente del sitio meridional de Kamihun (De Boer et al. 1977), lo que contrasta con la relativa abundancia de tiestos descubiertos en la provincia de Pastaza. Una contemporaneidad parcial o total de este conjunto con el Pastaza-Kamihun es posible por varias razones. La más significativa es, sin duda, la existencia de rasgos compartidos en algunos tiestos. Uno de ellos (Figura 4.10) presenta incisiones, achurados y muescas bajo el labio, más bien típicas de la decoración Pastaza-Kamihun, pero estas decoraciones se encuentran en un recipiente con borde evertido, labio relativamente grueso y con presencia de engobe rojo, lo cual lo aproxima claramente al conjunto inciso-exciso con engobe rojo.

\section{Cerámica con engobe rojo pulido (57 tiestos)}

Esta cerámica fina con engobe rojo intenso, descrita por Porras en su publicación, ha pasado completamente desapercibida, sin duda debido a que las

FIG. 4 - Tiestos incisos-excisos con engobe rojo

1. Borde de cuenco muy abierto;

2. Borde de cuenco con paredes casi verticales;

3. Borde de cuenco cerrado;

4. Borde de cuenco; el motivo espiral contiene incisiones y excisiones;

5. Borde de cuenco, con motivo triangular inciso y exciso;

6. Borde de cuenco conteniendo un motivo de una figura triangular compuesta realizada con excisiones e incisiones;

7. Borde de recipiente. Contiene un motivo circular realizado por incisión, dispuesto en medallón bajo el borde. Nótese la ranura que corre a lo largo del labio y que apareció en 3 tiestos de la colección;

8. Borde de cuenco, contiene motivos subtriangulares achurados incisos dispuestos perpendicularmente;

9. Borde de recipiente « rojo inciso », proveniente de la región de Macuma. Dibujo del autor según Echeverría et al. (1996, Fig. 6);

10. Borde de cuenco con características Pastaza-Kamihun como los motivos incisos y otras pertenecientes al conjunto inciso-exciso con engobe rojo (forma evertida y presencia de engobe anaranjado). 
ilustraciones están en blanco y negro. Es original dentro del contexto amazónico y no tiene relación con el conjunto inciso-exciso con engobe rojo, tanto desde el punto de vista de las pastas como del color del engobe. Esta cerámica se caracteriza en particular por tener dos pastas diferentes, las cuales encontramos en 54 de los 57 tiestos. La primera se caracteriza por un color gris con núcleo negro; es de textura arcillosa y tiene poco desgrasante; lleva partículas rosadas y blancas; contiene asperezas al interior; está representada por 41 fragmentos. La otra se diferencia por un color que va del anaranjado al beige. Se reconocen partículas blanquecinas y brillantes inferiores a $1 \mathrm{~mm}$, observándose también granos negros. Este grupo está constituido por 13 tiestos cuyo espesor se sitúa entre 0,3 y $0,5 \mathrm{~cm}$.

De los 57 tiestos, 53 son fragmentos de cuencos. Tres parecen provenir de formas cerradas, desgraciadamente no identificables. Pocos bordes pueden ser orientados y medidos. Se cuenta con tres cuencos abiertos (Figura 5.1), y un cuenco ligeramente cerrado. Los diámetros se sitúan entre 25 y $17 \mathrm{~cm}$. Los tiestos están recubiertos por un engobe rojo pulido, siempre brillante al exterior y a veces al interior. Así mismo, 12 tiestos llevan restos de pintura negra al interior y/o al exterior, y sus motivos son geométricos formando entrecruzamientos de líneas pintadas acompañadas de triángulos (Figura 5.2). Por otro lado, 3 fragmentos revelan la existencia de incisiones muy finas al interior. Desgraciadamente el material está fragmentado lo que no permite decir mucho. A causa de la fragmentación del material, es todavía prematuro el definir; a partir de los tiestos que llevan pintura negra o incisiones, una variante específica dentro del conjunto de cerámica con engobe rojo pulido.

Esta cerámica corresponde a lo que Porras llamó « Pastaza Roja ». Pero él reconoció únicamente un tipo de pasta y no hace alusión a los motivos decorativos. Estos últimos, totalmente desconocidos en los datos arqueológicos amazónicos, nos podrían hacer recordar motivos de las regiones andina y amazónica en épocas tardías (por ejemplo, en la vertiente oriental, el Cosanga-Píllaro, en Porras 1975b; o la cerámica roja reportada en el Norte de la Amazonía ecuatoriana: véase Aguilera, Arellano y Carrera 2003, pp. 148, 178-180). Por otro lado, un tiesto que parece pertenecer a este conjunto, pero sin pintura, se encuentra en el Museo Arqueológico y Etnográfico de Puyo y proviene de una zona más septentrional a la del sitio Huasaga, probablemente de las riveras del río Pastaza.

\section{Cerámica con motivos triangulares impresos (17 tiestos)}

Este conjunto de tiestos se caracteriza esencialmente por dos pastas que se ven representadas por 11 y 4 tiestos respectivamente. La primera, de textura arcillosa y de color anaranjado a marrón, cuenta con numerosos granos grises, blancos y brillantes de tamaño muy variable, generalmente superior a 1 o $2 \mathrm{~mm}$. Sin embargo, el desgrasante es inferior al $50 \%$ del volumen de la pasta que conlleva 

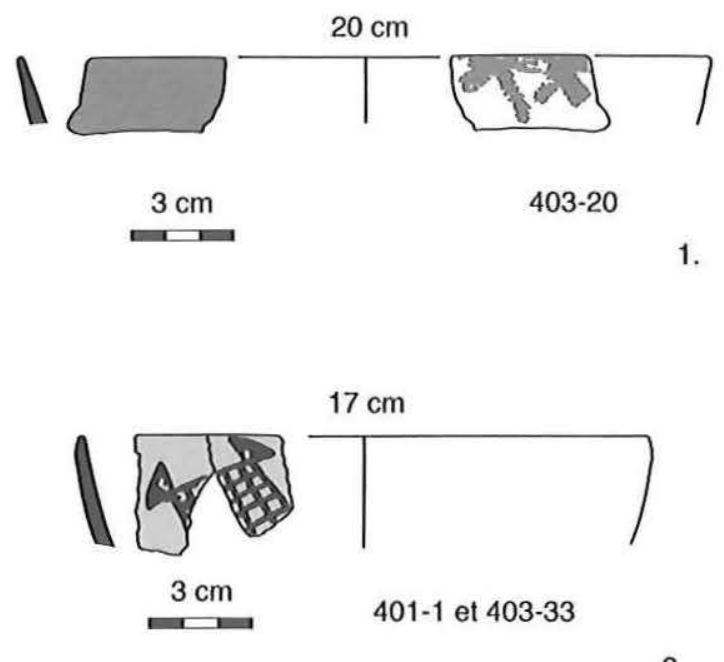

2.

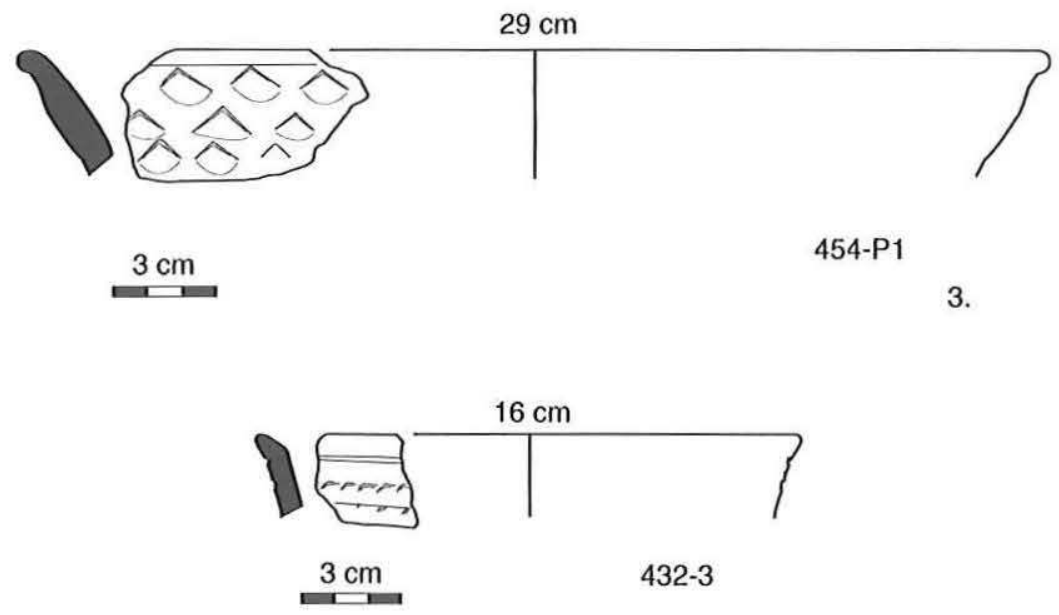

4.

FIG. 5 - Tiestos con engobe rojo pulido, con motivos triangulares impresos, y motivos de impresiones lineales

1. Borde de cuenco perteneciente al conjunto de engobe rojo pulido;

2. Borde de cuenco perteneciente al conjunto de engobe rojo pulido, contiene motivos geométricos pintados de negro;

3. Cuello de olla evertido perteneciente al conjunto de motivos triangulares impresos;

4. Cuello de olla evertido perteneciente al conjunto de motivos de impresiones lineales. 
generalmente asperezas. La segunda pasta parece ser una variante de la primera, pero más gruesa en la medida en que el desgrasante alcanza o sobrepasa el $50 \%$ del volumen de la pasta. El espesor promedio de los tiestos se sitúa entre 0,7 y más de $1 \mathrm{~cm}$.

Hay dos tiestos de formas abiertas (cuencos) y 15 de formas cerradas (ollas). Dos bordes pueden ser orientados y medidos; el uno proviene probablemente de un cuenco o de una escudilla de $20 \mathrm{~cm}$ de diámetro, y la otra corresponde tal vez a un cuello evertido de una olla donde la abertura mide $29 \mathrm{~cm}$ de diámetro (Figura 5.3).

La decoración corresponde a formas triangulares que llegan a un máximo de $1,5 \mathrm{~cm}$ de la base a la punta, impresas regularmente sobre la superficie todavía fresca del recipiente, tal vez por medio de una astilla de madera tallada en forma triangular.

Este tipo no aparece en el sitio de Pumpuentsa al oeste de Huasaga, ni en la colección del museo del Puyo.

\section{Cerámica con impresiones lineales (18 tiestos)}

Es un conjunto que, tanto por las técnicas decorativas como por algunas pastas, parece relacionarse con el conjunto precedente. Esto podría significar que formaron parte de una misma tradición. Tres pastas agrupan a 15 de los 18 tiestos. La más representada corresponde a la segunda pasta del conjunto de motivos triangulares impresos ( 8 tiestos). La siguiente corresponde a la primera pasta del mismo conjunto cerámico (4 tiestos). Finalmente, la última pasta, de color gris a anaranjado, es homogénea y no contiene ninguna aspereza. Ésta comprende únicamente 3 tiestos: contiene abundante desgrasante el cual mide alrededor de $1 \mathrm{~mm}$ de grosor; sin embargo, se pueden encontrar partículas más gruesas, de color blanco y gris, e incluso pequeños guijarros.

El espesor promedio de los tiestos se sitúa entre 0,4 y $1 \mathrm{~cm}$.

De la totalidad de los tiestos, 3 provienen de formas abiertas y 15 de formas cerradas del tipo olla. Sólo un borde está lo suficientemente bien conservado como para ser orientado y medido; éste corresponde a un cuello de olla ligeramente evertido, con apertura de $16 \mathrm{~cm}$ de diámetro (Figura 5.4).

La decoración se caracteriza por la impresión de puntos (óvalos o semitriángulos) sobre la pasta fresca. Es difícil identificar el objeto usado para realizar estas impresiones, cordel u otro instrumento (vegetal, concha, etc.).

\section{Cerámica perteneciente al horizonte Corrugado (203 tiestos)}

En esta cerámica se han encontrado más de siete pastas diferentes, entre las cuales se han identificado dos de las pastas del conjunto con motivos triangulares impresos. La primera abarca un total de 85 tiestos, en tanto que la variante gruesa 
totaliza 90. Por otro lado, también aparece una pasta de color leonado que parece ser una variante de las dos precedentes, pero que se distingue por su fineza. Su desgrasante está constituido por partículas inferiores a $1 \mathrm{~mm}$ que le confieren un aspecto arenoso al tacto. Ésta aparece en 19 tiestos. Las demás pastas son residuales puesto que corresponden a 1 ó 2 tiestos.

Globalmente, el espesor de los tiestos es muy variable: entre 0,4 y $1,3 \mathrm{~cm}$.

De un total de 203 tiestos, 13 provienen de cuencos y 190 de ollas. Entre los bordes que pueden ser orientados y medidos se cuenta con 3 bordes de cuencos bien abiertos, de entre 16 y $28 \mathrm{~cm}$ de diámetro (Figura 6.1), y 39 bordes de ollas. Entre estos últimos se puede señalar un grupo de ollas con cuello derecho o evertido (Figura 6.2 y 6.3), muy a menudo con borde doblado, y un grupo de ollas sin cuello pero con labio fuertemente evertido (Figura 6.4). No se encontraron asas.

La decoración corrugada se caracteriza por la utilización de acordelados más o menos reconocibles al exterior del recipiente, lo cual produce un efecto de ondulación horizontal en la superficie cerámica. En las ollas se sitúa generalmente al nivel del cuello, mientras que en los cuencos recubre la totalidad de la superficie exterior. En la colección de Porras, se reconocen variaciones y asociaciones con otras técnicas decorativas. Las modalidades decorativas más frecuentes son las siguientes: corrugados regulares (es decir con bandas de arcillas horizontales, continuas y del mismo tamaño) y otros irregulares (es decir con bandas de arcillas más o menos horizontales, discontinuas y de tamaño irregular). La mayoría presenta bandas de arcilla de 0,5 a $2 \mathrm{~cm}$ de alto. La obliteración parcial de las bandas por presión sobre la pasta fresca es frecuente, así como los pellizcados regulares de los acordelados frescos para que el efecto visual de ondulación sea tanto vertical como horizontal (Figura 6.5). Se encuentran también otras impresiones, casi siempre realizadas con uñas. Algunas modalidades decorativas son raras: hay dos ejemplos de utilización de pintura beige, y algunos tiestos tienen impresiones digitales perpendiculares con relación a los acordelados, realizadas en la pasta fresca. También hay que señalar diez fragmentos de ollas casi idénticos: son cuellos reducidos al mínimo o inexistentes, con borde fuertemente evertido. La decoración se caracteriza por impresiones de uñas simples o entrecruzadas, dispuestas en líneas (Figura 6.6). Los tiestos provienen en su totalidad de un solo sondeo (sondeo 1), a una profundidad comprendida entre 0 y $20 \mathrm{~cm}$, lo que podría indicar una ocupación tardía, incluso moderna, posterior al supuesto nuevo depósito del material por acción del río.

Este conjunto, tanto por las pastas como por las formas y las decoraciones, es extremadamente diverso, lo cual explica por qué actúa como una tradición abarcando la totalidad de la Amazonía ecuatoriana por más de 1000 años. El corrugado está presente en todas las provincias amazónicas del Ecuador, en parte de Perú y Colombia, y en la provincia serrana de Loja (Guffroy ed. 1987), la cual fue poblada al final del período prehispánico por miembros del grupo lingüístico 

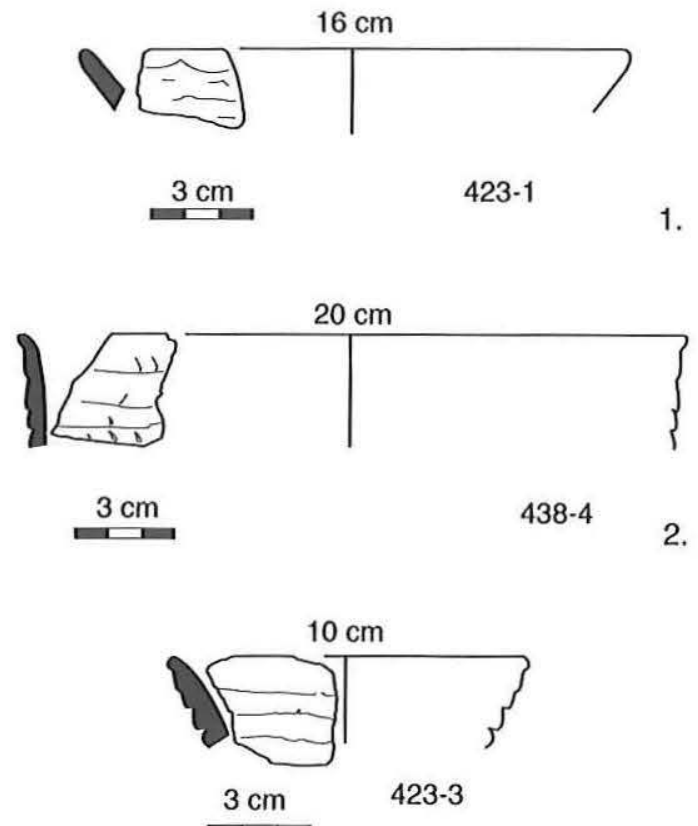

3.
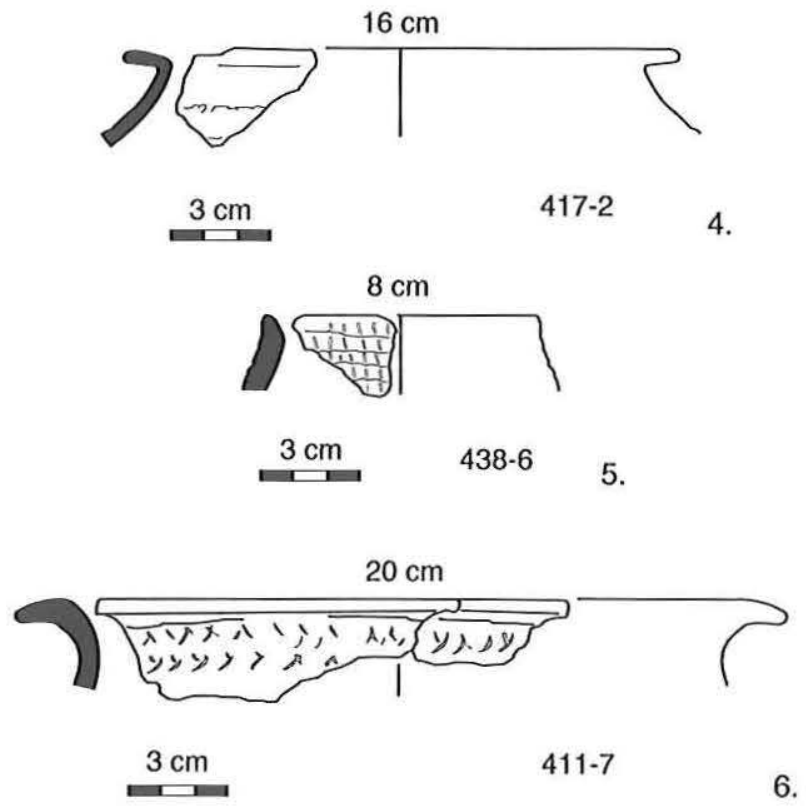

296

FIG. 6 - Conjunto corrugado 
jíbaro, probablemente originarios de la Amazonía. El conjunto Corrugado aparece tardíamente al Este de los Andes, donde remplaza los desarrollos culturales anteriores: en el valle del río Upano (Rostain 1999), en Zamora-Chinchipe (Guffroy, Valdez y Saulieu 2003), y en la provincia de Loja (Guffroy ed.1987; Guffroy 2004), el horizonte corrugado, según las dataciones de ${ }^{14} \mathrm{C}$, aparece a partir del siglo viII de nuestra era. Sin embargo, el corrugado presente en la colección Huasaga se destaca por tener un aspecto bastante grueso y más pobre que en otras regiones. Las formas son bastante sencillas (es decir comparables a una sola figura geométrica); no hay formas carenadas o muy compuestas, y las decoraciones no son finas. Además, no se encuentran asas, ni apliqués en bandas serpentiformes como las que se encuentran en las provincias de Loja y ZamoraChinchipe (Guffroy ed. 1987; Guffroy 2004; Guffroy y Valdez 2001). Así mismo, no observamos ornamentaciones muy cargadas en base a muescas, pellizcados e impresiones, ni la diversidad de formas que aparecen en el corrugado del Norte de la Amazonía ecuatoriana (como por ejemplo en la provincia de Sucumbíos, ver Aguilera, Arellano y Carrera 2003). Este corrugado es bastante simple, de tal manera que parece haber sido de uso cotidiano.

\section{Conclusiones provisionales}

La colección de Huasaga constituida por Pedro Porras permite elaborar un primer balance de los materiales cerámicos presentes en el Sur de la Amazonía ecuatoriana, zona todavía poco conocida de los arqueólogos (Tabla 2).

\begin{tabular}{|l|l|l|l|l|}
\hline \multicolumn{1}{|c|}{ Conjuntos cerámicos } & \multicolumn{1}{c|}{$\begin{array}{c}\text { Cuencos } \\
\text { y/o tecomates }\end{array}$} & $\begin{array}{c}\text { Ollas con } \\
\text { o/sin cuello }\end{array}$ & $\begin{array}{c}\text { No } \\
\text { identificables }\end{array}$ & Total \\
\hline Cerámica Pastaza-Kamihun & 740 & 0 & 0 & 740 \\
\hline Cerámica perteneciente al horizonte Corrugado & 13 & 190 & 0 & 203 \\
\hline Cerámica incisa y excisa con engobe rojo & 81 & 1 & 7 & 89 \\
\hline Cerámica con engobe rojo pulido & 53 & 3 & 1 & 57 \\
\hline Cerámica con impresiones lineales & 3 & 15 & 0 & 18 \\
\hline Cerámica con motivos triangulares impresos & 2 & 15 & 0 & 17 \\
\hline
\end{tabular}

TABLA 2 - Resumen de los conjuntos cerámicos presentes en la colección Pastaza de Porras, en orden creciente de cantidad.

FIG. 6 - Conjunto corrugado

1. Borde de cuenco con decoración corrugada obliterada;

2. Cuello de olla recto, con decoración corrugada acompañada por impresiones de uñas;

3. Cuello de olla evertido, con decoración corrugada;

4. Borde de olla sin cuello fuertemente evertido, con decoración corrugada;

5 . Borde de olla sin cuello, con decoración corrugada, pellizcado regularmente;

6. Borde de olla fuertemente evertido, con decoración constituida por impresiones entrecruzadas de uñas. 
Lo que impresiona en primer lugar es la gran variedad del material, aunque todavía no sabemos si esto se debe a la cronología, a una diversidad de poblamiento en un momento dado, o a otros fenómenos, tales como intercambios culturales. No se puede asegurar que esta diversidad representa una sola fase cultural como lo sostenía Porras. Además es muy probable que el PastazaKamihun no tenga ningún vínculo con el horizonte Corrugado. En la colección de Huasaga, si bien los materiales están mezclados, las pastas son extremadamente diferentes. Por otro lado, se debe insistir en la decoración y en el acabado del Pastaza-Kamihun y del Corrugado que son muy distintos. Además, se debe tomar en cuenta que dos sitios que contienen tiestos Pastaza-Kamihun (Pumpuentsa y Kamihun) no presentan tiestos corrugados.

Aunque aún no podemos aseverar que el Pastaza-Kamihun es anterior al Corrugado, un conjunto de índices lo sugiere. Primeramente, el corrugado perduró hasta nuestros días en algunas poblaciones que pertenecen al grupo lingüístico jíbaro y quichua de la región, y no el Pastaza-Kamihun. En segundo lugar, las dataciones de Stephen Athens (Athens 1986), pese a los problemas que tienen y que hemos subrayado en la introducción, sitúan el material Pastaza hacia el fin del siglo viI y el principio del siglo viII de nuestra era (Tabla 1). Ahora bien, a pesar de que hoy en día se presume que el Corrugado no apareció en la Amazonía ecuatoriana hasta el siglo vIII de nuestra era, queda mucho por hacer para confirmarlo.

Por otra parte, es posible, por razones estilísticas y por la presencia de un tiesto con carácteres mezclados, que el Pastaza-Kamihun haya sido aproximadamente contemporáneo del conjunto cerámico inciso y exciso con engobe rojo. En cuanto al material corrugado, algunos tiestos comparten las mismas pastas que los tiestos de los conjuntos con motivos triangulares impresos y con impresiones lineales. Por lo tanto, podríamos decir que estos tres conjuntos formaron parte de una misma tradición.

Desde el punto de vista de las formas, el Pastaza-Kamihun se caracteriza por su poca variedad así como por la total ausencia de ollas. Estas características las comparte con el conjunto cerámico inciso y exciso con engobe rojo, aunque no totalmente; recordamos en particular que en este último conjunto detectamos un fragmento de olla. Los otros conjuntos cerámicos descritos poseen formas tanto abiertas como cerradas. Insistimos sobre el hecho de que otro contraste proporciona un argumento más para separar el Pastaza-Kamihun del Corrugado: en efecto, en el primero encontramos una fineza y riqueza decorativa ausente en el segundo, mientras que en el segundo existen cuencos bastante gruesos finalmente, hay diferencias importantes en lo referente a las pastas.

Las comparaciones entre las decoraciones nos permiten también manejar la hipótesis según la cual el Pastaza-Kamihun constituye una variante de un conjunto Pastaza más general, caracterizado por incisiones geométricas hechas sobre pasta fresca, el cual habría tenido por lo menos dos variantes más: una en 
la parte occidental (un Pastaza-Kamihun con motivos figurativos y achurado zonal), y la otra localizada cerca de Copataza (Figura 1).

Finalmente vale la pena señalar un punto sorprendente, aunque puede estar relacionado con la escasez de investigaciones: la ausencia, en Huasaga, en Pumpuentsa y en Kamihun, de tiestos traídos de o influenciados por los grupos Upano los cuales están situados al oeste de la provincia, al pie de la cordillera de los Andes. Sabemos que esta cerámica ha sido objeto de intercambios comerciales, ya que tiestos Upano han sido descubiertos en la Sierra central del Ecuador (Bruhns et al. 1994; Rostocker 1996; Rostain 1999). En el mismo sentido, la poca diversidad de las formas cerámicas del Huasaga contrasta con su profusión en el Upano (Porras 1987; Rostocker 1996, 2005). ¿Se trataría de una ausencia de contactos? ¿Cada grupo habría privilegiado regiones diferentes para sus intercambios (el Upano con las tierras altas, el Pastaza con la Amazonía fluvial)? ¿O se trataría de un fenómeno de afirmación cultural fuerte, bastante frecuente en el período de Desarrollo regional en el Ecuador? Con seguridad un estudio más profundo del conjunto Pastaza a través de su cronología y de sus variantes permitiría aclarar el problema.

Agradecimientos: Mis agradecimientos van dirigidos a Patricio Moncayo, director del museo Weilbauer de la Universidad Católica de Quito, por su acogida y su apertura, a Guadalupe Cruz, Susana Mogollón, Francisco Valdez y Jean Guffroy. Agradezco también a Alfonsina Andrade y Alden Yépez; sin ellos, este trabajo de revisión no habría tenido resultados. Este texto ha sido traducido gracias a Juan Sarrade, Estanislao Pazmiño y Lydia Andrés.

\section{REFERENCIAS BIBLIOGRAFICAS}

Aguilera María, Jorge Arellano y Juan Carrera

2003 Cuyabeno ancestral, Corporación Simbioe, Quito.

Athens Stephen

1984 «Pumpuentsa: un sitio arqueológico cerca del río Macuma en el Oriente ecuatoriano », Miscelánea antropológica ecuatoriana, 4, pp. 129-140.

1986 «The site of Pumpuentsa and the Pastaza phase in Southeastern Lowland Ecuador ", Ñawpa Pacha, 24, pp. 111-124.

Brunhns Karen O., James H. Burton y Arthur G. Rostoker

1994 "La cerámica incisa en franjas rojas: evidencias de intercambio entre la Sierra y el Oriente en el Formativo Tardío del Ecuador ", in Izumi Shimada (ed.), Tecnología y organización de la producción de cerámica prehispánica en los Andes, Pontificia Universidad Católica del Perú, Fondo Editorial, Lima, pp. 53-66. 
DE BoER Warren

1975 Two ceramic collections from Rio Huasaga, Northern Peru: their place in the prehistory of the Upper Amazon, Queens College Laboratory of Archeology, CUNY, New York.

De Boer Warren, Eric Ross, Jane Ross y Mary Veale

1977 "Two ceramic collections from Rio Huasaga, Northern Peru: their place in the prehistory of the Upper Amazon ", El Dorado, 2 (2), pp. 1-11, University of Northern Colorado.

ECHEVERRÍA José

1981 Glosario arqueológico, Instituto Otavaleño de Antropología, Otavalo/ Ediciones Pucará, Lima.

Echeverría José, Inge Schejllerup y Pablo Morales

1996 Informe de observación de asentamientos antiguos en Oyacachi-El Chaco (provincia de Napo), Canelos-Chapeta (provincia de Pastaza), MacumaMutiats y área de San Luis de Ininkes (provincia de Morona Santiago), región amazónica ecuatoriana, Informe INPC, Quito.

\section{GuFroy Jean}

2004 Catamayo precolombino: investigaciones arqueológicas en la provincia de Loja (Ecuador), Universidad técnica particular de Loja, Banco central del Ecuador, Loja/Instituto francés de estudios andinos, Lima/Institut de recherche pour le développement, Orléans.

GuFfroy Jean (ed.)

1987 Loja préhispanique: recherches archéologiques dans les Andes méridionales de l'Équateur, Éditions Recherche sur les civilisations, Paris.

GUFFroy Jean y Francisco VALDEZ

2001 Resultados de la etapa de reconocimiento (1999-2000) y proyecto de investigación arqueológica (2001-2004) en la provincia de Zamora-Chinchipe, Informe preliminar INPC, Quito.

GuFFroy Jean, Francisco VALdeZ y Geoffroy de SAULIeU

2003 "Découverte d'une ancienne civilisation en Amazonie occidentale ", Fiche d'actualité scientifique de l'IRD, 177, www.ird.fr/fr/actualites/fiches/ 2003/fiche177.htm

Lathrap Donald Ward, Donald Collier y Helen Chandra

1975 Ancient Ecuador: Culture, clay and creativity 3000-300 B.C. [Catalogue of an exhibit organized by Field Museum of Natural History April 18-August 5 1975], Field Museum of Natural History, Chicago.

LUMBRERAS Luis G.

2005 "Un formativo sin cerámica y cerámica pre-formativa », in Jorge Hidalgo (ed.), Actas del $51^{\circ}$ congreso internacional de americanistas, $14-18$ de julio 2003, Universidad de Chile, Santiago del Chile. 
Meggers Betty J.

1987 "The early history of man in Amazonia ", in Timothy C. Whitmore y Ghillean T. Prance (eds), Biogeography and quaternary history in tropical America, Clarendon Press, Oxford, pp. 151-174.

Porras Pedro

1975a "Fase Pastaza. El formativo en el oriente ecuatoriano », Revista de la Universidad Católica, III (10), Quito.

1975b Fase Cosanga: estudios cientificos sobre el Oriente ecuatoriano, Centro de Publicaciones de la Pontificia Universidad Católica del Ecuador, Quito.

1987a Investigaciones arqueológicas en las faldas del Sangay: tradición upano, Centro de Investigaciones Arqueológicas, Quito.

1987b Mamual de arqueología ecuatoriana, Centro de Investigaciones Arqueológicas, Quito.

RAMPÓN Lino

1959 "Sitio arqueológico F.P. », Cuadernos de Investigaciones Científicas, 1, Arqueología, Quito.

\section{Rostain Stéphen}

1999 «Secuencia arqueológica en montículos del valle del Upano en la Amazonía ecuatoriana », Bulletin de l'Institut francais d'études andines, 28 (1), pp. 53-89.

RosTOKER Arthur G.

1996 Archaeological assemblage from Eastern Ecuador, San Francisco State University, Treganza Anthropology Museum Papers 19, San Francisco CA.

2005 Dimension of prehistoric human occupation in the Southern Ecuadorian Oriente, Dissertation submitted to the graduate Faculty of Anthropology in partial fulfillment of the requirements for the degree of Doctor of Philosophy, CUNY, New York.

Valdez Francisco, Jean Gufrroy, Geoffroy de Saulieu, Julio Hurtado y Alexandra YÉPEZ

2005 «Découverte d'un site cérémoniel formatif sur le versant oriental des Andes 》, Palévol 4 (4), pp. 369-374.

Zıolkowski Mariusz, Mieczyslaw Pazdur, Andrej KrZanowskı y Adam MichCZynski 1994 Andes: radiocarbon database for Bolivia, Ecuador and Peru, Andean Archaeological Mission of the Institute of Archaeology, Warsaw University, Warszawa/Gliwice Radiocarbon Laboratory of the Institute of Physics, Silesian Technical University, Gliwice. 